\title{
Piezotronic effect at Schottky barrier of a metal-Zno single crystal interface
}

Cite as: J. Appl. Phys. 121, 155701 (2017); https://doi.org/10.1063/1.4981243

Submitted: 06 February 2017 • Accepted: 05 April 2017 • Published Online: 18 April 2017

Peter Keil, Till Frömling, (iD) Andreas Klein, et al.
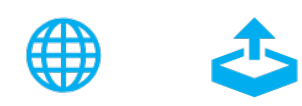

View Online

\section{ARTICLES YOU MAY BE INTERESTED IN}

A comprehensive review of $\mathrm{ZnO}$ materials and devices

Journal of Applied Physics 98, 041301 (2005); https://doi.org/10.1063/1.1992666

$\mathrm{BaTiO}_{3}$-based piezoelectrics: Fundamentals, current status, and perspectives Applied Physics Reviews 4, 041305 (2017); https://doi.org/10.1063/1.4990046

ZnO Schottky barriers and Ohmic contacts

Journal of Applied Physics 109, 121301 (2011); https://doi.org/10.1063/1.3581173

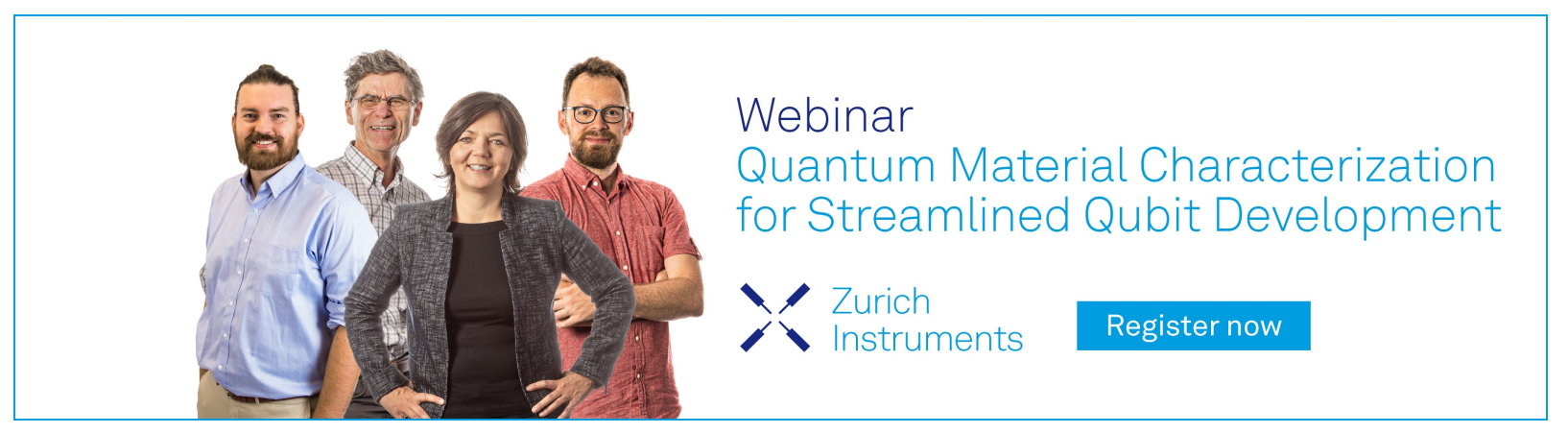




\title{
Piezotronic effect at Schottky barrier of a metal-ZnO single crystal interface
}

\author{
Peter Keil, Till Frömling, Andreas Klein, Jürgen Rödel, and Nikola Novak ${ }^{\mathrm{a})}$ \\ Institute of Materials Science, Technische Universität Darmstadt, Alarich-Weiss Straße 2, 64287 Darmstadt, \\ Germany
}

(Received 6 February 2017; accepted 5 April 2017; published online 18 April 2017)

\begin{abstract}
$\mathrm{ZnO}$ is considered as one of the most promising semiconductor materials for future applications based on the piezotronic effect. Intense studies on $\mathrm{ZnO}$ nanowires had been carried out to understand the modulation of the Schottky barrier height at the metal $\mathrm{ZnO}$ interface via piezoelectricity. However, an experimental investigation on bulk $\mathrm{ZnO}$ single crystals and a fundamental comparison of the modification of the barrier height determined experimentally and theoretically are still missing. Therefore, an adjustment of the electrostatic potential barrier height at metal- $\mathrm{ZnO}$ single crystal interfaces due to stress induced piezoelectric charges was conducted, using both $\mathrm{O}$ - and $\mathrm{Zn}$-terminated surfaces. In-situ stress dependent impedance and current-voltage measurements were utilized to extract the electrical properties of the potential barrier and to determine the reduction of the barrier height. The decrease of the interface resistance and increase of the capacitance reveal the presence of stress induced piezoelectric charges. The experimentally evaluated reduction of the barrier height reveals a moderate change of about $9 \mathrm{meV}$ at $70 \mathrm{MPa}$ and supports prior work on metal-ZnO nanowires. This change was found to be in good agreement with theoretical calculations based on the imperfect screening model if a thickness of the interface layer is assumed to be $\sim 2 \AA$ A Published by AIP Publishing.

[http://dx.doi.org/10.1063/1.4981243]
\end{abstract}

\section{INTRODUCTION}

The adjustment of the height of electrostatic potential barriers represents one of the most intriguing and challenging tasks since the development of semiconductor based electronics. In the classical description of the metalsemiconductor junction, the electrostatic potential barrier is created due to the difference of the electron affinity between metal and semiconductor (Schottky-Mott rule). ${ }^{1}$ However, the experimental results on metal- $\mathrm{ZnO}$ junctions demonstrated that the barrier height often is independent of the metal and was rationalized by Fermi level pinning. ${ }^{2-4}$ Fermi level pinning is related to the presence of interface trap states which form interface dipole moments with charges at the metal surface and define the barrier height of the junction. ${ }^{5}$ Cowley and Sze introduced a phenomenological model which uses the Fermi level pinning approach combined with the presence of a finite interfacial layer thickness, $\delta_{\text {int }}$, between metal and semiconductor. Trapped electrons at the interface build up an electric field which affects the electron transport across the barrier. To adjust the potential barrier height, the charge density and distribution at the interface have to be modified. The electric field at the semiconductor interface can be tuned by introducing a thin dopant layer at the semiconductor surface via ion implantation or by the presence of piezoelectric charges. ${ }^{6-9}$ By varying the doping concentration and profile, the barrier height can be adjusted as required. Recently, an approach utilizing piezoelectric properties of semiconductors to dynamically modify the potential barrier height raised considerable interest. ${ }^{10-12}$ This

\footnotetext{
a) Author to whom correspondence should be addressed. Electronic mail: novak@ceramics.tu-darmstadt.de
}

concept relies on reduction or increase of the potential barrier height via stress generated piezoelectric charges at the interface. The interaction between stress induced piezoelectric charges and electrostatic potential barrier is known as piezotronic effect. Due to the exponential relationship between the barrier height and current density through a Schottky contact, small changes of the barrier height drive a change in current density by several orders of magnitude. Hence, switching between a conductive and non-conductive state, i.e., between on and off state with stress opens the possibility to develop innovative devices for sensor applications or piezoelectric diodes. ${ }^{11}$ Most materials of interest for piezotronic applications are of wurtzite type crystal structure such as $\mathrm{GaN}$, InN, and $\mathrm{ZnO}$. With their non-centrosymmetric hexagonal structure, they exhibit piezoelectric and semiconducting properties and have been considered as promising piezotronic materials. ${ }^{8,12-15}$ Due to its high piezoelectric coefficient, $\mathrm{ZnO}$ came into the focus of research, mostly as a metal- $\mathrm{ZnO}$ nanowire heterostructure or in its polycrystalline form. ${ }^{14,16-20}$ Charge transport is determined by the barrier height of the Schottky contact and can be described by thermionic emission theory. ${ }^{1}$ If an external mechanical stress is applied to the piezotronic material, piezoelectric charges are generated at the metal-semiconductor interface, which subsequently changes the barrier height and hence the charge transport across the barrier. ${ }^{11}$ For an n-type Schottky contact with piezoelectrically induced positive charge, the Schottky barrier height will decrease, while negative charge increases the barrier height. ${ }^{11}$ However, rather moderate changes of the barrier height with stress, i.e., $\sim 7 \mathrm{meV}$ at $300 \mathrm{~K}$ at a stress of $\sim 1.4 \mathrm{GPa}$ and strain of $\sim 1 \%$ were observed in metal- $\mathrm{ZnO}$ nanowire structures. ${ }^{10}$ Small changes in the barrier height 
were attributed to screening effects due to the high conductivity of the $\mathrm{ZnO}$ nanowires but yielded a high gauge factor quantifying the modification in resistivity with strain. ${ }^{10,21}$ Despite increasing interest in the piezotronic effect on Schottky barriers at the metal-ZnO interface, fundamental investigations on bulk single crystals are missing. The advantage of bulk studies is that the applied stress can be precisely defined, whereas in the case of $\mathrm{ZnO}$-nanowires the applied strain is calculated and depends on the diameter of the nanowire and adhesion between the nanowire and substrate. In addition, a direct comparison between the theoretical calculation and experimentally observed variation of the barrier height is lacking.

To investigate the reduction of the barrier height at metal-ZnO single crystal interfaces, Schottky contacts were prepared both on $\mathrm{Zn}$ - and O-terminated surfaces of the crystals. Current-voltage and impedance measurements under different uniaxial mechanical stress states quantified the effect of stress on the height of the Schottky barrier. The theoretically predicted reduction of the barrier height was computed using an imperfect screening model, which was applied before to rationalize the effect of spontaneous polarization on the Schottky barrier height at ferroelectric metal interfaces. $^{22,23}$ The comparison of experimentally determined and theoretically calculated barrier height reveals the performance of $\mathrm{ZnO}$ as a piezotronic material and provides insight into the underlying mechanisms.

\section{EXPERIMENTAL}

$\mathrm{ZnO}$ single crystals (Alineason Materials Technology GmbH, Frankfurt, Germany) with dimensions of about $5 \times 5 \times 5 \mathrm{~mm}^{3}$ were 6 -site mechano-chemically polished by the manufacturer. The conductivity and carrier concentration mobility of the wafers were $0.03 \mathrm{~S} / \mathrm{cm}$ and $5.4 \times 10^{15} \mathrm{~cm}^{-3}$ from room temperature Hall effect measurements using the van der Pauw method. Prior to Schottky barrier formation, samples were cleaned with acetone, ethanol, and de-ionized water in an ultrasonic bath. Schottky contacts were prepared either on the Zn-terminated surface (0001) or O-terminated surface $(000 \overline{1})$ of the crystal by silver deposition. Metallic silver electrodes of a thickness of about $150 \mathrm{~nm}$ were prepared by sputter deposition followed by annealing in air for $2 \mathrm{~h}$ at $400{ }^{\circ} \mathrm{C}$. The annealing step oxidizes the silver electrode and promotes the rectifying behavior of the contact. ${ }^{24}$ Ohmic contacts on the opposite side were provided by $\mathrm{Al} / \mathrm{Au}$ electrodes, also via sputter deposition (Emitech K950X, Quorum Technologies Ltd, Ashford, Kent, UK). All electrical measurements under different externally applied mechanical fields ranging from $5 \mathrm{MPa}$ to $70 \mathrm{MPa}$ were conducted inside a screw-driven load frame Zwick Z010 (Zwick GmbH \& Co. KG, Ulm, Germany). While the load was applied perpendicular to the c-axis of the crystal, the electrical measurements were conducted parallel to the c-axis (see Figure 1). Hence, a direct application of stress on the metal electrodes was avoided and side effects related to the deformation of the electrode were omitted.

All current-voltage $(I-V)$ measurements were recorded in a range between $-1 \mathrm{~V}$ and $1 \mathrm{~V}$ using a Keithley 2450

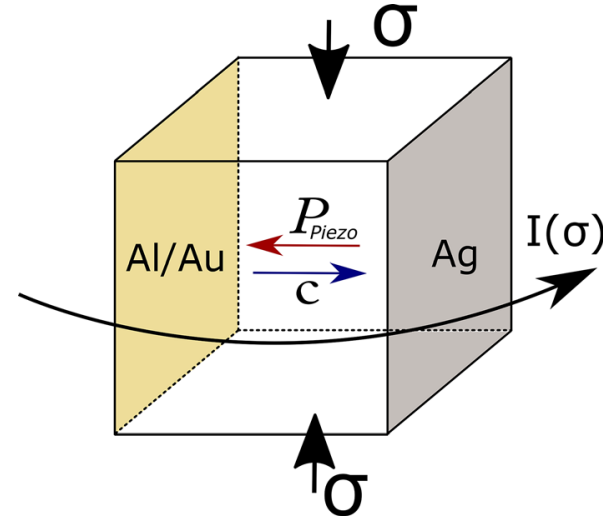

FIG. 1. Schematic representation of measurement arrangement. Mechanical stress is applied perpendicular to c-axis, while electrical measurement is performed in parallel direction with c-axis of the crystal.

SourceMeter (Keithley Instruments, Inc., Cleveland, OH, USA). The impedance spectra were recorded with an impedance analyzer (Novocontrol Technologies GmbH \& Co. KG, Montabaur, Germany) in a frequency range between $1 \mathrm{~Hz}$ and $1 \mathrm{MHz}$. The maximum amplitude of the ac voltage was set to $V_{r m s}=0.015 \mathrm{~V}$ to ensure that the junction properties are not measured in the upturn region of the current voltage characteristics.

\section{RESULTS AND DISCUSSION}

Uniaxial compressive stress applied perpendicular to the c-axes of the crystal induces positive piezoelectric charge on the Zn-terminated surface, which should lead to a reduction of the barrier height at the corresponding Schottky barrier. Figure 2(a) presents the characteristic $I-V$ curves as a function of compressive stress in a semi-logarithmic plot. With
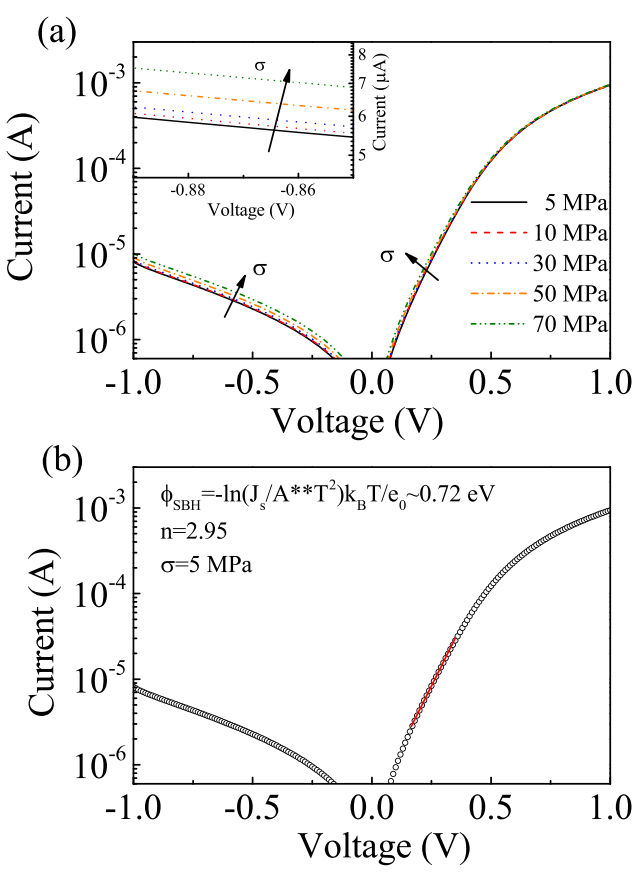

FIG. 2. (a) Stress dependent current-voltage characteristic of Schottky contact on the Zn-terminated surface of $\mathrm{ZnO}$ single crystal. (b) Current-voltage characteristic of the same Schottky contact at $5 \mathrm{MPa}$ compressive stress. 
increasing stress, a small increase of the current can be observed in reverse and forward biasing (see the inset in Figure 2(a)). The $I-V$ response obtained at $5 \mathrm{MPa}$ (see Figure 2(b)) is treated as the initial state where the effect of mechanical stress was neglected. Following the thermionic analysis, the barrier height of the metal-semiconductor contact was found to be $\phi_{S H B} \sim 0.72 \mathrm{eV}$ with an ideality factor of $n=2.95$. $^{1}$ The ideality factor describes the deviation from the pure thermionic transport $(n=1)$ and represents a measure of the quality of the Schottky contact. The deviation of the ideality factor from unity has been ascribed to different factors such as an inhomogeneous distribution of barrier heights, tunneling current, and generation/recombination currents. ${ }^{25,26}$ Due to the large area of the Schottky contact $\left(A=25 \mathrm{~mm}^{2}\right)$ utilized in this work, a spatial variation of the barrier height is expected to lead to the deviation from the ideal thermionic process. ${ }^{25}$

To verify that the stress dependent measurements are not an artifact of the setup or due to the modification of the surface, the same measurements were repeated for a sample with the Schottky contact deposited on the O-terminated surface (Figure 3(a)). Due to the piezoelectric effect, a negative charge is generated on the O-polar surface. Hence, an increase of the barrier height and a decrease of the current are expected. With increasing stress, a moderate decrease in current can be observed under reverse bias (see the inset in Figure 3(a)). Nevertheless, the fundamental concept that the change in polarity changes the direction of the piezotronic effect can be confirmed, which in turn verifies that the increasing conductivity measured on the $\mathrm{Zn}$-terminated surface is a consequence of the piezotronic effect. From the $I-V$ measurement at $5 \mathrm{MPa}$, the barrier height and ideality factor of $\phi_{S H B} \sim 0.72 \mathrm{eV}$ and $n=3.23$ were determined (see
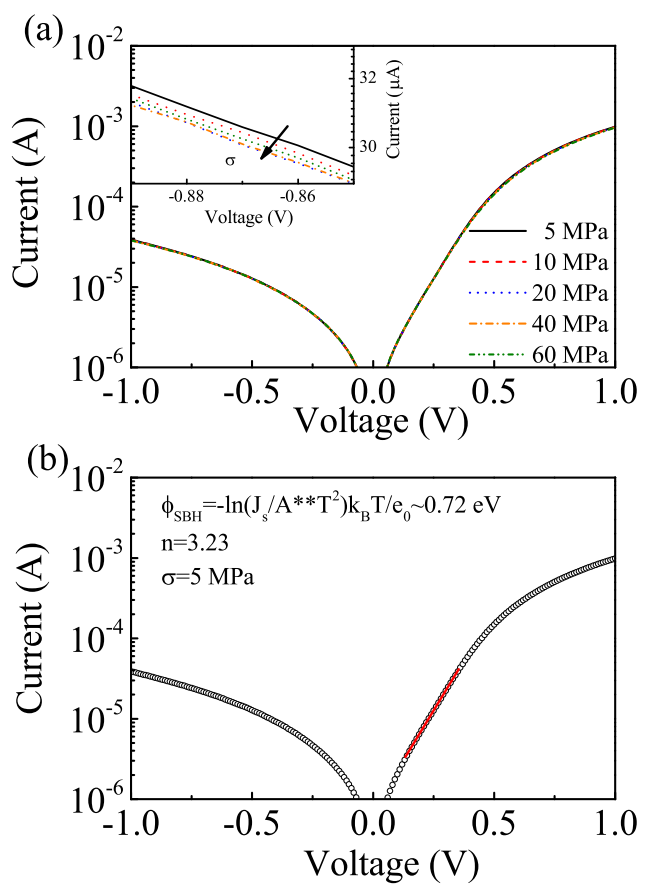

FIG. 3. (a) Stress dependent current-voltage characteristic of Schottky contact on the O-terminated surface of $\mathrm{ZnO}$ single crystal. (b) Current-voltage characteristic of the same Schottky contact at $5 \mathrm{MPa}$ compressive stress.
Figure 3(b)). Work by Allen et al. verified that surface polarity using $\mathrm{Ag}$ electrodes has no effect on the barrier height. ${ }^{27}$ In addition, the barrier heights of $0.72 \mathrm{eV}$ can be related to the presence of the oxygen vacancies which were considered as possible defect states at the interface responsible for the pinning of the Fermi level. ${ }^{28,29}$ The higher reverse current of the contact deposited on the O-terminated surface can be explained by the increased ideality factor which reflects the inhomogeneous distribution of the barrier height. ${ }^{25}$ This may also be the reason for the smaller changes observed under applied stress.

For applications, the switching between the nonconductive and conductive state, i.e., a reduction of the barrier height is of particular interest. Therefore, detailed studies in the following focused on Zn-terminated surfaces. To elucidate the effect of the induced piezoelectric charges on the electrical properties of the Schottky barrier on the Zn-terminated surface, in-situ impedance measurements at different stresses have been conducted. Figure 4(a) depicts the Nyquist plot of impedance spectra obtained at different uniaxial mechanical stresses. The low-frequency value of the real component of impedance decreases with increasing stress. Due to the high intrinsic conductivity of the bulk $\mathrm{ZnO}$ single crystal, it is expected that the low frequency impedance response of the fabricated heterostructure is governed by electrical properties of the Schottky contact and that only electrical properties of the bulk and the Ohmic contact contribute to the impedance response at higher frequencies. Therefore, the observed decrease of the real part of impedance at low frequencies can be attributed to the decrease in the resistance of the Schottky contact. It is expected that a lower barrier height increases the leakage current; hence, the resistance of the interface decreases. A similar behavior of
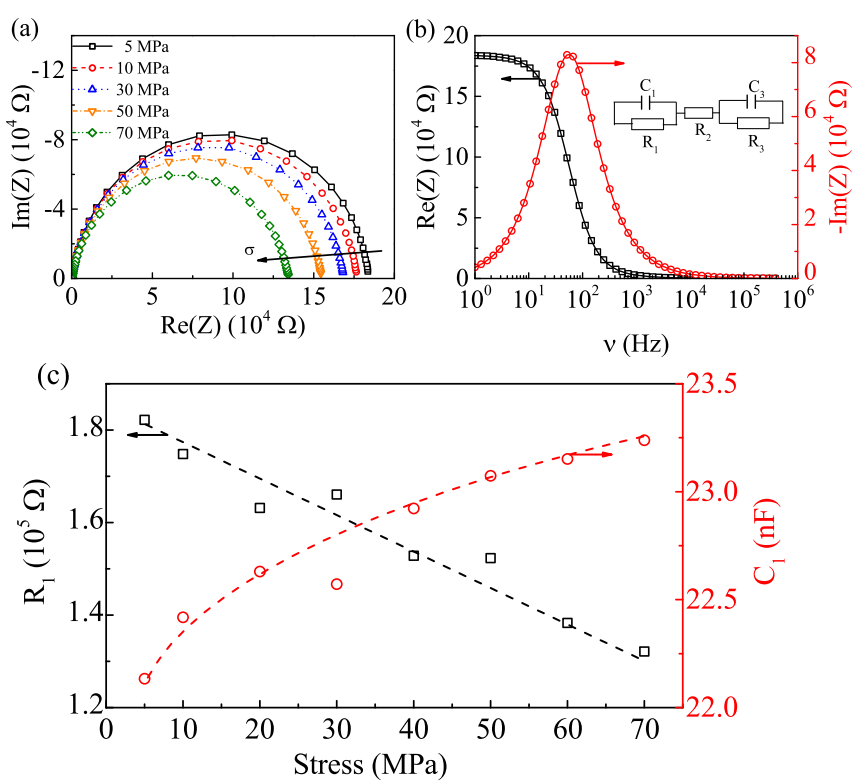

FIG. 4. (a) Complex plane plot of the $\mathrm{ZnO}$ single crystal with Ag-based Schottky contact on the Zn-terminated surface under increasing mechanical stress. (b) Spectroscopic plot of the measured real and imaginary part of impedance $\mathrm{Z}$ for $5 \mathrm{MPa}$ of compressive stress, lines represent the simulated results based on the depicted equivalent circuit. (c) Extracted resistance and capacitance for the depletion region of the Schottky contact. 
impedance was observed at Schottky contacts formed to $\mathrm{Si}$, measured under various dc voltages applied in forward direction. $^{30}$

The electrical properties of the Schottky contact were extracted from the equivalent circuit analysis of the impedance data. ${ }^{31}$ Here, the equivalent circuit consists of three parts representing Schottky contact, $\mathrm{ZnO}$ bulk material, and Ohmic contact. The network of capacitors and resistors connected in series and parallel, used to describe the impedance response, is represented schematically in Figure 4(b). The first part consists of $R_{1}$ and $C_{1}$ in parallel, which represents the resistance and capacitance of the Schottky contact, the second part is represented by $R_{2}$ and describes the resistance of the bulk. The third part quantifies the Ohmic contact and is represented by resistance $R_{3}$ and $C_{3}$ connected in parallel. During the fitting process, both capacitances were represented by a constant phase element (CPE), which accounts for a possible defectiveness of the capacitance behavior of both metal-semiconductor interfaces. The empirical impedance function of the CPE is $Z_{C P E}=A(j \omega)^{-\alpha}$, with $\alpha=1$ describing an ideal capacitor and $\alpha=0$ describing a pure resistor. ${ }^{31}$ The $\alpha$-value of the Schottky contact was above 0.93 for all mechanical stresses, while the $\alpha$-value of the Ohmic contact was in the range of 0.72 . For a perfectly Ohmic contact, one would expect an $\alpha=0$ representing a perfect resistive behavior, still the observed intermediate value of the Ohmic contact may have two reasons: (i) aluminum which was used to fabricate the Ohmic contact may not be perfectly Ohmic, ${ }^{32}$ and (ii) following the theory for the piezotronic effect a space charge layer can build up on the site of the Ohmic contact when a mechanical stress is applied. ${ }^{11}$ The measured data of the real and the imaginary part of impedance as a function of frequency were simultaneously fitted with Equations (1) and (2) which quantify the impedance response of the equivalent circuit

$$
Z^{\prime}=\frac{R_{1}}{1+C_{1}^{2}+\omega^{2} R_{1}^{2}}+R_{2}+\frac{R_{3}}{1+C_{3}^{2} \omega^{2} R_{3}^{2}}
$$

and

$$
Z^{\prime \prime}=-\frac{C_{1} \omega}{C_{1}^{2} \omega^{2}+\frac{1}{R_{1}^{2}}}-\frac{C_{3} \omega}{C_{3}^{2} \omega^{2}+\frac{1}{R_{3}^{2}}} .
$$

An example of the equivalent circuit fit, which well describes the impedance response of the fabricated $\mathrm{ZnO}$ heterostructure, is provided in Figure 4(b). From the fits, resistance and capacitance of the space charge region, resistance of the bulk $\mathrm{ZnO}$ single crystal, and Ohmic contact were extracted. The resistance of the bulk $\mathrm{ZnO}$ single crystal and Ohmic contact were found to be constant over the whole range of applied mechanical stress with an average value of $\sim 65 \Omega$ and $\sim 1500 \Omega$, respectively. The impedance value of the bulk is in good agreement with the resistance obtained from Hall measurements $(67 \Omega)$. In Figure 4(c), the variation of resistance and capacitance obtained for the depletion region of the Schottky contact is demonstrated as a function of mechanical stress. The barrier height determines the current transport through the Schottky junction. The interface resistance $R_{1}$ decreases by $\sim 27 \%$, which corresponds to the $\sim 27 \%$ increase of the reverse bias current (obtained from $I-V$ curves). The reduction of the resistance is accompanied by a simultaneous increase in capacitance $C_{1}$. The capacitance of the depletion layer is given by ${ }^{1}$

$$
C \equiv \frac{\partial Q}{\partial V}=\sqrt{\frac{e_{0} \varepsilon_{0} \varepsilon_{s} N_{D}}{2\left(\phi_{S B H}-\xi-\frac{k_{B} T}{e_{0}}\right)}},
$$

where $e_{0}$ is the elementary charge, $N_{D}$ is the donor concentration, $k_{B}$ is the Boltzmann constant, $\xi$ is the energy difference between the conduction band and Fermi level, $T$ is the temperature, and $\varepsilon_{s}$ and $\varepsilon_{0}$ are permittivity of the semiconductor and free space, respectively. Assuming that the donor concentration remains constant, the increase in capacitance is caused by the reduction of the barrier height with mechanical stress.

The stress dependent $I-V$ and impedance measurements clearly demonstrate the effect of mechanical stress on the Schottky barrier properties in terms of reduction of barrier height and interface resistance as well as an increase of interface capacitance.

From the stress dependent $I-V$ curves highlighted in Figure 2(a), the barrier height was determined as a function of uniaxial stress (Figure 5). A rather moderate change in barrier height, $\Delta \phi_{S B H} \sim 9 \mathrm{meV}$, was achieved if the material was stressed with a uniaxial mechanical field of $70 \mathrm{MPa}$. The small change in the barrier height is comparable with the results reported for the metal- $\mathrm{ZnO}$ nanowire $(7 \mathrm{meV})$, but it needs to be stressed that in the current work the reduction is achieved at much smaller mechanical stress (70 MPa vs. $\sim 1.4 \mathrm{GPa}) .{ }^{10}$ As mentioned above, the small changes in the barrier height in $\mathrm{ZnO}$ nanowires were attributed to internal screening of piezoelectric charges. ${ }^{21}$

Theoretical calculations were undertaken to underpin the experimental performance of metal- $\mathrm{ZnO}$ interfaces. The line in Figure 5 represents the theoretically calculated reduction of the barrier height considering the imperfect screening model. ${ }^{22,23}$ Stengel et al. used this model to describe the

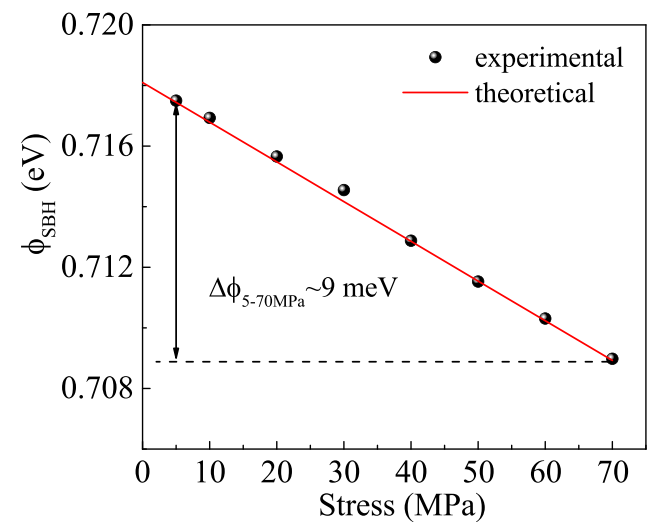

FIG. 5. Reduction of barrier height as a function of applied stress as determined from $I-V$ measurement. The line represents the calculated value of the barrier height as a function of stress using the imperfect screening model. 
effect of the spontaneous polarization on the barrier height at metal-ferroelectric interfaces. The model is based on the assumption that a thin vacuum interface layer between metal and ferroelectric material is present similar as considered for classical metal-semiconductor junctions with the presence of trap states. ${ }^{2,22}$ Considering the stress induced charges represented by piezoelectric polarization, $P_{\text {piezo }}$, the barrier height, $\phi_{S H B}$, can be calculated as a function of stress ${ }^{11,22,23}$

$$
\phi_{S H B}=\phi_{S H B}^{0} \pm e_{0} \frac{P_{\text {piezo }} \delta_{\text {int }}}{2 \varepsilon_{0}} \phi .
$$

Here, $\phi_{S H B}^{0} \sim 0.72 \mathrm{eV}$ is the barrier height determined at $5 \mathrm{MPa}$. The piezoelectric polarization can be calculated by using the relation between the polarization, mechanical stress, $\sigma_{1}$, and piezoelectric coefficient, $d_{31}$, i.e., $P_{\text {piezo }}=$ $d_{31} \sigma_{1}$ with a piezoelectric coefficient of $d_{31}=-5.8 \mathrm{pC} / \mathrm{N}^{33}$ Hence, the induced piezoelectric charge at maximum applied stress of $70 \mathrm{MPa}$ is $0.0406 \mu \mathrm{C} / \mathrm{cm}^{2}$. The experimentally obtained reduction of the barrier height can be well described with Equation (4) if the thickness of the interface layer is assumed to be $\delta_{i n t} \sim 2 \AA$. The interface layer thickness of $2 \AA$ can be favorably contrasted to the literature where the interface thickness between metal and semiconductor is assumed to be few Angstroms. In the case of metalferroelectric interfaces, it is even smaller., ${ }^{2,9}$ The good agreement between the calculated and experimentally obtained results questions viability of the internal screening mechanism, to explain the limited piezotronic performance. From Equation (4), it is apparent that the change of the barrier height strongly depends on the polarization at the interface and the thickness of the interface layer. The piezoelectric polarization can be increased by increasing the external mechanical stress. However, at stresses above $100 \mathrm{MPa}$ the probability of mechanical damage is enhanced. Therefore, to decrease the operational mechanical stress, the thickness of the interface layer needs to be increased to obtain a higher variation of barrier height. Assuming an extension of $\delta_{\text {int }}$ by ten times $(\sim 2 \mathrm{~nm})$ e.g., by the introduction of an artificial "dead layer," the barrier reduction under the same mechanical condition could be dramatically increased $(\sim 90 \mathrm{meV})$. Hence, the current in reverse bias would increase for about two orders of magnitude due to its exponential dependency on the barrier height. ${ }^{1}$ The question remains open, if the piezotronic effect at other interfaces could be tailored to yield considerably higher changes in the piezotronic effect and thereby larger modulations in resistivity.

\section{CONCLUSIONS}

Schottky barriers at $\mathrm{Zn}$-terminated $\mathrm{ZnO}$ single crystal surfaces feature a decreased potential barrier height and increased current under uniaxial compressive stress with Oterminated surfaces revealing the opposite effect. The magnitude of change in the potential barrier height in $\mathrm{Zn}$ terminated interfaces of $9 \mathrm{meV}$ supports work on the piezotronic effect at Schottky contacts in metal- $\mathrm{ZnO}$ nanowires with similar numerical results. A model based on imperfect screening rationalizes the experimental results and suggests the existence of an interface layer with a thickness of $2 \AA$. Succinct understanding of the electrical interface conditions may open pathways for tailoring Schottky contacts with an improved resistance ratio and strain sensing capability.

\section{ACKNOWLEDGMENTS}

This work was financed by the Deutsche Forschungsgemeinschaft (DFG) under Project No. RO954/28-1.

${ }^{1}$ S. M. Sze, Physics of Semiconductor Devices, 2nd Edition (John Wiley \& Sons Inc., New York, 1981).

${ }^{2}$ A. M. Cowley and S. M. Sze, J. Appl. Phys. 36(10), 3212-3220 (1965).

${ }^{3}$ K. Ip, G. T. Thaler, H. Yang, S. Y. Han, Y. Li, D. P. Norton, S. J. Pearton, S. Jang, and F. Ren, J. Cryst. Growth 287(1), 149-156 (2006).

${ }^{4}$ U. Ozgur, Y. I. Alivov, C. Liu, A. Teke, M. A. Reshchikov, S. Dogan, V. Avrutin, S. J. Cho, and H. Morkoc, J. Appl. Phys. 98(4), 041301-041103 (2005).

${ }^{5}$ J. Bardeen, Phys. Rev. 71(10), 717-727 (1947).

${ }^{6}$ J. M. Shannon, Appl. Phys. Lett. 24(8), 369-371 (1974).

${ }^{7}$ J. M. Shannon, Solid-State Electron. 19(6), 537-543 (1976).

${ }^{8}$ O. Ambacher, J. Smart, J. R. Shealy, N. G. Weimann, K. Chu, M. Murphy, W. J. Schaff, L. F. Eastman, R. Dimitrov, L. Wittmer, M. Stutzmann, W. Rieger, and J. Hilsenbeck, J. Appl. Phys. 85(6), 3222-3233 (1999).

${ }^{9}$ M. Stengel, D. Vanderbilt, and N. A. Spaldin, Nat. Mater. 8(5), 392-397 (2009).

${ }^{10}$ J. Zhou, Y. Gu, P. Fei, W. Mai, Y. Gao, R. Yang, G. Bao, and Z. L. Wang, Nano Lett. 8(9), 3035-3040 (2008).

${ }^{11}$ Y. Zhang, Y. Liu, and Z. L. Wang, Adv. Mater. 23(27), 3004-3013 (2011).

${ }^{12}$ R. Yu, L. Dong, C. Pan, S. Niu, H. Liu, W. Liu, S. Chua, D. Chi, and Z. L. Wang, Adv. Mater. 24(26), 3532-3537 (2012).

${ }^{13}$ O. Ambacher, B. Foutz, J. Smart, J. R. Shealy, N. G. Weimann, K. Chu, M. Murphy, A. J. Sierakowski, W. J. Schaff, L. F. Eastman, R. Dimitrov, A. Mitchell, and M. Stutzmann, J. Appl. Phys. 87(1), 334-344 (2000).

${ }^{14}$ Z. L. Wang, Nano Today 5(6), 540-552 (2010).

${ }^{15}$ N.-J. Ku, C.-H. Wang, J.-H. Huang, H.-C. Fang, P.-C. Huang, and C.-P. Liu, Adv. Mater. 25(6), 861-866 (2013).

${ }^{16}$ J. H. He, C. L. Hsin, J. Liu, L. J. Chen, and Z. L. Wang, Adv. Mater. 19(6), 781-784 (2007).

${ }^{17}$ Z. L. Wang and J. Song, Science 312(5771), 242-246 (2006).

${ }^{18}$ R. Baraki, N. Novak, T. Frömling, T. Granzow, and J. Rödel, Appl. Phys. Lett. 105(11), 111604 (2014).

${ }^{19}$ R. Baraki, N. Novak, M. Hofstätter, P. Supancic, J. Rödel, and T. Frömling, J. Appl. Phys. 118(8), 085703 (2015).

${ }^{20}$ N. Raidl, P. Supancic, R. Danzer, and M. Hofstätter, Adv. Mater. 27(12), 2031-2035 (2015).

${ }^{21}$ Y. Hu, B. D. B. Klein, Y. Su, S. Niu, Y. Liu, and Z. L. Wang, Nano Lett. 13(11), 5026-5032 (2013).

${ }^{22}$ M. Stengel, P. Aguado-Puente, N. A. Spaldin, and J. Junquera, Phys. Rev. B 83(23), 235112 (2011).

${ }^{23}$ L. Pintilie and M. Alexe, J. Appl. Phys. 98(12), 124103 (2005).

${ }^{24}$ M. W. Allen, R. J. Mendelsberg, R. J. Reeves, and S. M. Durbin, Appl. Phys. Lett. 94(10), 103508 (2009).

${ }^{25}$ J. H. Werner and H. H. Guttler, J. Appl. Phys. 69(3), 1522-1533 (1991).

${ }^{26}$ R. T. Tung, Phys. Rev. B 45(23), 13509-13523 (1992).

${ }^{27}$ M. W. Allen, M. M. Alkaisi, and S. M. Durbin, Appl. Phys. Lett. 89(10), 103520 (2006).

${ }^{28}$ M. W. Allen and S. M. Durbin, Appl. Phys. Lett. 92(12), 122110 (2008).

${ }^{29}$ S. Siol, J. C. Hellmann, S. D. Tilley, M. Graetzel, J. Morasch, J. Deuermeier, W. Jaegermann, and A. Klein, ACS Appl. Mater. Interfaces 8(33), 21824-21831 (2016).

${ }^{30}$ C. Yim, N. McEvoy, and G. S. Duesberg, Appl. Phys. Lett. 103(19), 193106 (2013).

${ }^{31}$ E. Barsoukov and J. Ross Macdonald, Impedance Spectroscopy Theory, Experiment, and Applications, 2nd edition ed. (John Wiley \& Sons, Inc., Hoboken, New Jersey, 2005).

${ }^{32}$ H.-K. Kim, K.-K. Kim, S.-J. Park, T.-Y. Seong, and I. Adesida, J. Appl. Phys. 94(6), 4225-4227 (2003).

${ }^{33}$ A. R. Hutson, Phys. Rev. Lett. 4(10), 505-507 (1960). 